\title{
Behaviour of topological defects of optical indicatrix orientation in cubic, hexagonal, trigonal and tetragonal crystals under conically distributed electric field. \\ 2. The electric field and the optical beam parallel to the principal crystallographic directions
}

\author{
Vasylkiv Yu., Kryvyy T., Skab I. and Vlokh R. \\ Vlokh Institute of Physical Optics, 23 Dragomanov Street, 79005 Lviv, Ukraine, \\ vlokh@ifo.lviv.ua
}

Received: 05.12 .2014

\begin{abstract}
We study the behaviour of topological defects (TDs) of optical indicatrix orientation under the conditions when electrooptic Pockels and Kerr nonlinearities coexist in the crystals belonging to cubic, hexagonal, trigonal and tetragonal systems and a conically shaped electric field is applied along the principal crystallographic and/or optic axes. The topological reactions accompanying the processes of birth, addition, division and annihilation of the TDs of optical indicatrix orientation are observed under electric field variations. It is shown that the conservation law for the strength of TDs holds true under these topological reactions and the behaviours of the TDs can be exhaustively described by four different scenarios.
\end{abstract}

Keywords: Pockels effect, Kerr effect, optical indicatrix, optical vortices, topological defects, topological reactions

PACS: $78.20 . J q, 42.50 . T x$

UDC: $535.5+537.228 .3+515.1$

\section{Introduction}

In the first part of the present study (see Ref. [1]) we have shown that, under application of a conically shaped electric field along the three-fold symmetry axis in single crystals belonging to the symmetry groups $\overline{4} 3 \mathrm{~m}$ and 23 of the cubic system, seven topological defects (TDs) of optical indicatrix orientation appear as a result of 'crossover' regime for the electrooptic Pockels and Kerr nonlinearities. An increase in the electric voltage gives birth to three pairs of TDs, which are referred conventionally to as $\mathrm{TD}_{1}$ and $\mathrm{TD}_{1}{ }_{1}, \mathrm{TD}_{2}$ and $\mathrm{TD}_{2}{ }_{2}$, and $\mathrm{TD}_{3}$ and $\mathrm{TD}_{3}$. In addition, a central $\mathrm{TD}, \mathrm{TD}_{0}$, also appears. These TDs are characterized by half-integer strengths and the opposite strength signs inside the pairs. Besides, it has been demonstrated that the defects $\operatorname{TD}_{1,2,3}$ move towards the centre of the beam cross section (i.e., towards the central defect $\mathrm{TD}_{0}$ ) with further increasing voltage, while the defects $\mathrm{TD}_{1,2,3}$ move out of the beam aperture. We have also revealed in the study [1] that the process described above is reversible, i.e. the pairs of TDs annihilate whenever the voltage decreases enough. The strength of the central defect $\mathrm{TD}_{0}$ does not change from $-1 / 2$ to 1 until the Pockels effect vanishes completely. Hence, the three TDs moving towards the central one never reach it with increasing voltage but only approach the central defect closely enough. The behaviour mentioned here strictly follows from the conservation law for the strength of TDs, although the total charge of the optical vortices caused by these defects is not conserved under the TD reactions [1].

Among different point symmetry groups describing optically isotropic (cubic) and optically uniaxial crystals, for which the Pockels and Kerr effects can coexist $(\overline{4} 3 \mathrm{~m}, 23, \overline{6} \mathrm{~m} 2, \overline{6}, \overline{4}, 3 \mathrm{~m}$, 
32, and 3 - see Ref. [2]), we have explored the behaviour of TDs of optical indicatrix orientation only for the first three symmetry groups $[1,2]$. Moreover, as follows from our further analysis, even for these groups the results have not been complete enough. This is why the present work is devoted to the further phenomenological and numerical analyses performed for the cases of crystals belonging to the cubic system. Besides, we will present the relevant analysis for the lowersymmetry groups $\overline{6}, \overline{4}, 3 \mathrm{~m}, 32$ and 3 .

\section{Results of analytical and numerical analyses}

In our analytical and numerical analyses, we have made use of a Jones matrix approach described in detail in Ref. [1]. The changes in the TD coordinates which occur with varying electric voltage are determined by solving numerically a system of nonlinear equations that follows from general formulae describing the orientation of optical indicatrix perturbed by the electric field due to the known Pockels and Kerr effects [1, 2].

Let us start from consideration of crystals of the point symmetry groups $\overline{4} 3 \mathrm{~m}$ and 23 , which are subjected to electric field directed along one of the principal crystallographic axes (e.g., [001]). Here we imply that a wide parallel light beam propagates along the same direction. For both the point groups, application of a homogeneous electric field along the [001] axis leads to the appearance of optical birefringence along this axis due to the linear Pockels effect. The optical indicatrix equation under the above conditions may be written as

$$
B_{1}^{0} X^{2}+B_{1}^{0} Y^{2}+B_{1}^{0} Z^{2}+2 r_{41} E_{3} X Y=1,
$$

where $\Delta B_{\lambda}=B_{\lambda}-B_{\lambda}^{0}=r_{\lambda k} E_{k}$ denote the changes in the optical impermeability coefficients arising under electric field components $E_{k}$ due to the linear electrooptic effect, $r_{\lambda k}=r_{i j k}$ are the components of a third-rank electrooptic Pockels tensor $(i j \leftrightarrow \lambda=1, \ldots 6)$, and $X, Y$ and $Z$ the Cartesian coordinate axes coinciding with the crystallographic axes $a, b$ and $c$, respectively.

Applying Eq. (1) to the crystals under interest, one readily obtains the optical birefringence and the angle of optical indicatrix rotation [3]:

$$
\Delta n_{X Y}=-n^{3} r_{41} E_{3}, \varsigma_{Z}= \pm 45 \mathrm{deg},
$$

where $n$ implies the unperturbed refractive index. As seen from Eqs. (2), a homogeneous electric field applied along the direction [001] induces the linear birefringence whenever the light propagates along the field direction. Hence, in the crystals of the symmetry groups $\overline{4} 3 \mathrm{~m}$ and 23 these directions do not remain optically isotropic. Then the TDs of optical indicatrix orientation cannot appear in the $X Y$ plane. Similarly, the conically shaped electric field also cannot create the TDs in the centre of the $X Y$ plane, i.e. $X=Y=0$ since the orientation of the optical indicatrix is strictly defined (see Eq. (2)). Notice in this respect that the appearance of TDs should imply an undefined orientation of the optical indicatrix in the TD core [4-6].

After accounting for the quadratic electroptic Kerr effect, the conical shape of the electric field and a smallness of the uniform cell in a crystal (for detailed explanations see Ref. [1]), one can rewrite Eqs. (2) for the group $\overline{4} 3 \mathrm{~m}$ as follows [7]:

$$
\begin{gathered}
\Delta n_{X Y}=-\frac{n^{3}}{2} \sqrt{\left(R_{11}-R_{12}\right)^{2}\left(E_{1}^{2}-E_{2}^{2}\right)+4\left(r_{41} E_{3}+R_{44} E_{1} E_{2}\right)^{2}}, \\
\tan 2 \zeta_{Z}=\frac{2\left(r_{41} E_{3}+R_{44} E_{1} E_{2}\right)}{\left(R_{11}-R_{12}\right)\left(E_{1}^{2}-E_{2}^{2}\right)} .
\end{gathered}
$$

where $E_{1}=k X, E_{2}=k Y$ and $E_{3}=k Z$ are the electric field components 
( $k=U Z / d\left(X^{2}+Y^{2}+Z^{2}\right)$, with $U$ being the electric voltage and $d$ the crystal sample thickness along $Z$ axis) and $R_{\lambda \mu}$ denote the Kerr tensor components $\left(R_{\lambda \mu}=R_{i j k l}\right.$ for $i j \leftrightarrow \lambda=1, \ldots 6 ; k l \leftrightarrow \mu=1,2,3$ and $R_{\lambda \mu}=2 R_{i j k l} \quad$ for $\left.i j \leftrightarrow \lambda=1, \ldots 6 ; k l \leftrightarrow \mu=4,5,6\right)$. In our numerical simulations we have used the following parameters: $r_{41}=10^{-12} \mathrm{~m} / \mathrm{V}$, $R_{11}=1.05 \times 10^{-18} \mathrm{~m}^{2} / \mathrm{V}^{2}, R_{12}=0.5 \times 10^{-18} \mathrm{~m}^{2} / \mathrm{V}^{2} \quad R_{44}=10^{-18} \mathrm{~m}^{2} / \mathrm{V}^{2}$, and $n=1.7$. The sample thickness along the [001] direction and the radius of the incident beam have been taken to be $5 \mathrm{~mm}$.

By solving the system of equations following from Eqs. (4), one gets

$$
\left\{\begin{array}{l}
r_{41} E_{3}+R_{44} E_{1} E_{2}=0 \\
\left(R_{11}-R_{12}\right)\left(E_{1}^{2}-E_{2}^{2}\right)=0
\end{array},\right.
$$

thus allowing to obtain the dependence of the coordinates of TDs on the voltage applied. As seen from Fig. 1a, two TDs, $\mathrm{TD}_{1}$ and $\mathrm{TD}_{2}$, with the strengths $+1 / 2$ appear if the conically shaped electrical field is applied along the [001] direction in the crystals belonging to the point group $\overline{4} 3 \mathrm{~m}$. These defects occupy the positions located at the bisectors of the $\pm X$ and $\mp Y$ axes. The phase difference in the TD cores is equal to zero (see Fig. 1b). These defects move towards the centre of the $X Y$ cross section of the beam with increasing voltage (see Fig. 1c), though they could reach the centre only in the hypothetic case when the Pockels effect vanishes. Then the system of
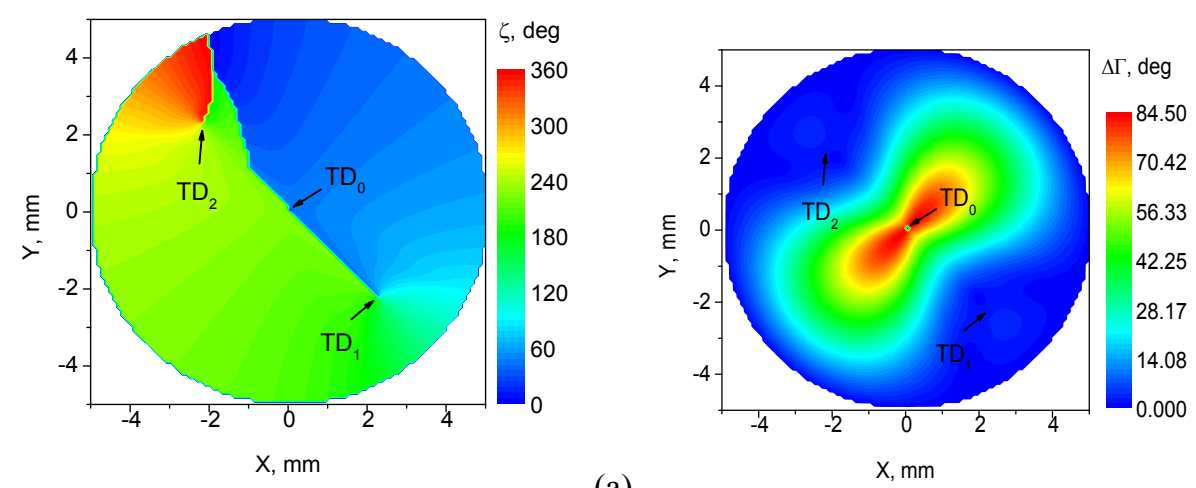

(a)

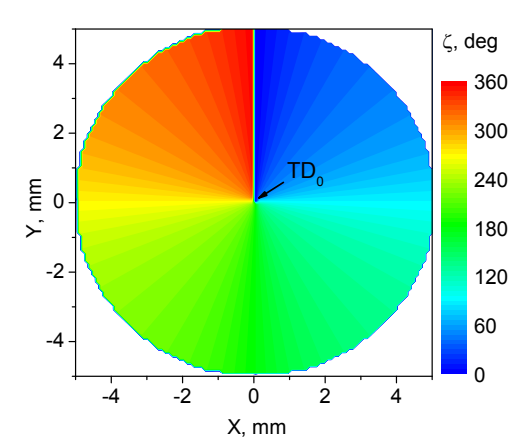

(c)

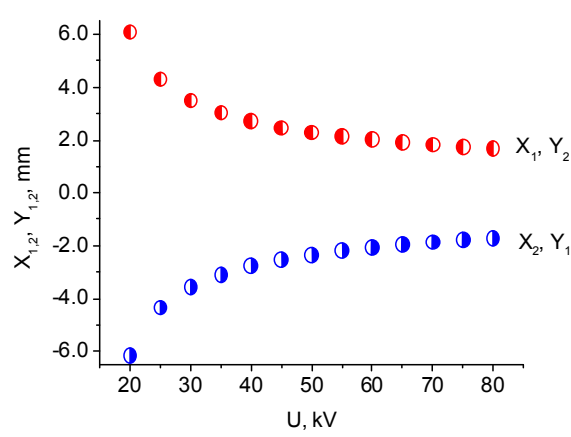

(d)

Fig. 1. TDs appearing in crystals of the point symmetry group $\overline{4} 3 \mathrm{~m}:$ (a) spatial map of the angle of optical indicatrix orientation under the electrooptic Pockels and Kerr effects $(U=30 \mathrm{kV})$, (b) map of the phase difference under the same conditions, (c) dependences of TD coordinates on the voltage applied, and (d) map of the angle of optical indicatrix orientation obtained in the case of pure electrooptic Kerr effect $(U=10 \mathrm{kV}) . X_{i}$ and $Y_{i}$ are coordinates of the defects $\operatorname{TD}_{i}$.

Ukr. J. Phys. Opt. 2015, Volume 16, Issue 1 
Eqs. (5) has a single solution at $X=Y=0$. Therefore only one defect $\left(\mathrm{TD}_{0}\right)$ with the strength +1 appears at these coordinates (see Fig. 1d) due to the Kerr effect (see Ref. [8]). In the latter case Eq. (4) reads as

$$
\tan 2 \zeta_{Z}=\frac{R_{44} E_{1} E_{2}}{\left(R_{11}-R_{12}\right)\left(E_{1}^{2}-E_{2}^{2}\right)}=\frac{R_{44}}{2\left(R_{11}-R_{12}\right)} \tan 2 \varphi,
$$

so that we have $\zeta_{Z} \approx \varphi$ at $R_{44} \approx 2\left(R_{11}-R_{12}\right)$ (here the notation $X=\rho \cos \varphi$ and $Y=\rho \sin \varphi$ is used, with $\varphi$ being the tracing polar angle). Hence, the topological reaction accompanying the process of adding of the defect strength $\left(p_{\mathrm{TD} 1}+p_{\mathrm{TD} 2}=p_{\mathrm{TD} 0}\right)$ may be written as $+1 / 2+1 / 2=+1$.

When passing to the tetragonal symmetry group $\overline{4}$, one should remind that this group represents a subgroup of the group $\overline{4} 3 \mathrm{~m}$, which is also characterized by the four-fold inversion symmetry axis. As a result, one can expect similar behaviours of the TDs for both the groups. For the group $\overline{4}$, the birefringence and the angle of optical indicatrix rotation within the homogeneous cell are given by the relations

$$
\begin{aligned}
& \Delta n_{X Y}=-\frac{n_{o}^{3}}{2} \sqrt{\begin{array}{l}
{\left[2 r_{13} E_{3}+\left(R_{11}-R_{12}\right)\left(E_{1}^{2}-E_{2}^{2}\right)+2 R_{16} E_{1} E_{2}\right]^{2}} \\
+4\left[r_{63} E_{3}+R_{61}\left(E_{1}^{2}-E_{2}^{2}\right)+R_{66} E_{1} E_{2}\right]^{2}
\end{array}}, \\
& \tan 2 \zeta_{Z}=\frac{2\left(r_{63} E_{3}+R_{61}\left(E_{1}^{2}-E_{2}^{2}\right)+R_{66} E_{1} E_{2}\right)}{2 r_{13} E_{3}+\left(R_{11}-R_{21}\right)\left(E_{1}^{2}-E_{2}^{2}\right)+2 R_{16} E_{1} E_{2}}
\end{aligned}
$$

Here we put the electrooptic coefficients to be equal to $r_{63}=0.5 \times 10^{-12} \mathrm{~m} / \mathrm{V}$, $r_{13}=1.2 \times 10^{-12} \mathrm{~m} / \mathrm{V}, \quad R_{66}=1.05 \times 10^{-18} \mathrm{~m}^{2} / \mathrm{V}^{2}, \quad R_{12}=0.1 \times 10^{-18} \mathrm{~m}^{2} / \mathrm{V}^{2}, \quad R_{11}=1 \times 10^{-18} \mathrm{~m}^{2} / \mathrm{V}^{2}$ and $R_{16}=0.5 \times 10^{-18} \mathrm{~m}^{2} / \mathrm{V}^{2}$, while the ordinary refractive index is $n_{\mathrm{o}}=1.7$. After solving numerically the system of equations

$$
\left\{\begin{array}{l}
r_{63} E_{3}+R_{61}\left(E_{1}^{2}-E_{2}^{2}\right)+R_{66} E_{1} E_{2}=0 \\
2 r_{13} E_{3}+\left(R_{11}-R_{21}\right)\left(E_{1}^{2}-E_{2}^{2}\right)+2 R_{16} E_{1} E_{2}=0
\end{array},\right.
$$

one obtains two solutions for the coordinates of the TDs (see Fig. 2a). Notice that the phase difference remains zero at these coordinates (see Fig. 2b). The dependences of the coordinates on the electric voltage are shown in Fig. 2c, d.

As seen from Fig. 2, the number of TDs observed in the crystals of the point group $\overline{4}$ and their behaviours under varying voltage are the same as those typical for the group $\overline{4} 3 \mathrm{~m}$. The only difference for the symmetry group $\overline{4}$ consists in the fact that the TDs do not occupy the diagonal positions between the $X$ and $Y$ axes. When the Pockels effect vanishes, only one defect with the unit strength remains in the centre of the $X Y$ cross section (see Fig. 2e). This is due to the topological reaction of adding defect strength.

Now let us proceed to the analysis for the cubic symmetry group 23. The induced birefringence and the angle of optical indicatrix rotation within the homogeneous cell for this group may be represented as

$$
\Delta n_{X Y}=-\frac{n^{3}}{2} \sqrt{\left[\left(R_{11}-R_{12}\right) E_{1}^{2}+\left(R_{12}-R_{11}\right) E_{2}^{2}+\left(R_{21}-R_{12}\right) E_{3}^{2}\right]^{2}+4\left(r_{41} E_{3}+R_{44} E_{1} E_{2}\right)^{2}}
$$



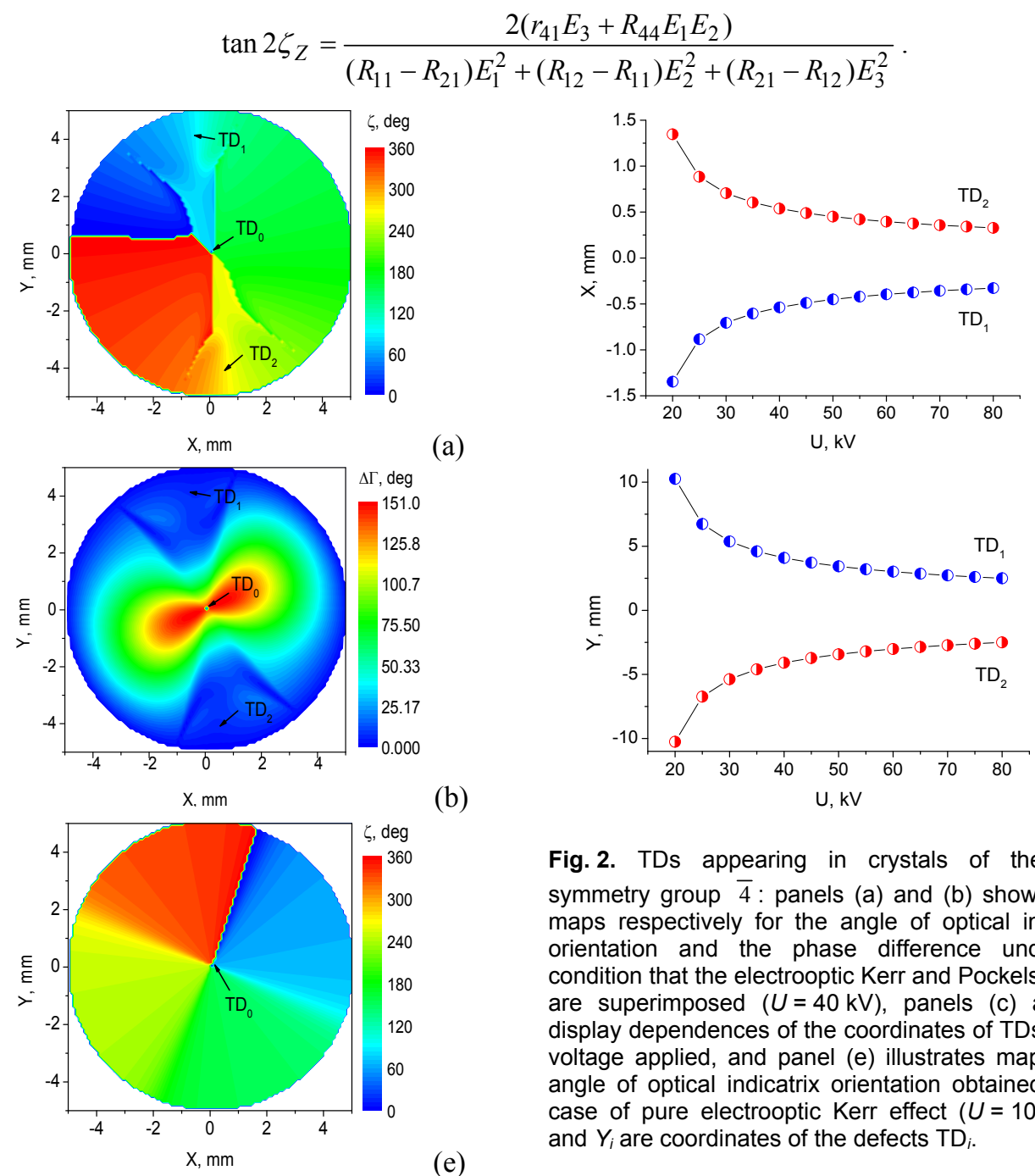

Fig. 2. TDs appearing in crystals of the point symmetry group $\overline{4}$ : panels (a) and (b) show spatial maps respectively for the angle of optical indicatrix orientation and the phase difference under the condition that the electrooptic Kerr and Pockels effects are superimposed $(U=40 \mathrm{kV})$, panels (c) and (d) display dependences of the coordinates of TDs on the voltage applied, and panel (e) illustrates map of the angle of optical indicatrix orientation obtained in the case of pure electrooptic Kerr effect $(U=10 \mathrm{kV}) . X_{i}$

(e) and $Y_{i}$ are coordinates of the defects $\mathrm{TD}_{i}$

The following quantitative values of the model parameters have been used: $r_{41}=1.5 \times 10^{-12} \mathrm{~m} / \mathrm{V}, R_{11}=1.2 \times 10^{-18} \mathrm{~m}^{2} / \mathrm{V}^{2}, R_{12}=0.5 \times 10^{-18} \mathrm{~m}^{2} / \mathrm{V}^{2}, R_{21}=0.2 \times 10^{-18} \mathrm{~m}^{2} / \mathrm{V}^{2}$, and $n=1.7$. By solving the system of equations

$$
\left\{\begin{array}{l}
r_{41} E_{3}+R_{44} E_{1} E_{2}=0 \\
\left(R_{11}-R_{21}\right) E_{1}^{2}+\left(R_{12}-R_{11}\right) E_{2}^{2}+\left(R_{21}-R_{12}\right) E_{3}^{2}=0
\end{array},\right.
$$

one can determine the TD coordinates and their dependences on the electric voltage. We have found that, in the crossover regime, two $\mathrm{TDs}\left(\mathrm{TD}_{1}\right.$ and $\left.\mathrm{TD}_{2}\right)$ are available for the group 23 , with the defect strengths equal to $+1 / 2$ (see Fig. 3a). These defects occupy the bisector positions between the $\pm X$ and $\mp Y$ axes. The phase difference is equal to zero at the points where the TD cores are located (see Fig. 3b). As seen from Fig. 4, the defects move towards the centre of the beam cross section with increasing voltage. When the Pockels effect vanishes, the defect $\mathrm{TD}_{0}$ with the strength +1 appears in the centre of the $X Y$ cross section (see Fig. 3c) as a result of topological reaction of adding $\mathrm{TD}_{1}$ and $\mathrm{TD}_{2}: p_{\mathrm{TD} 1}+p_{\mathrm{TD} 2}=+1 / 2+1 / 2=+1$. 

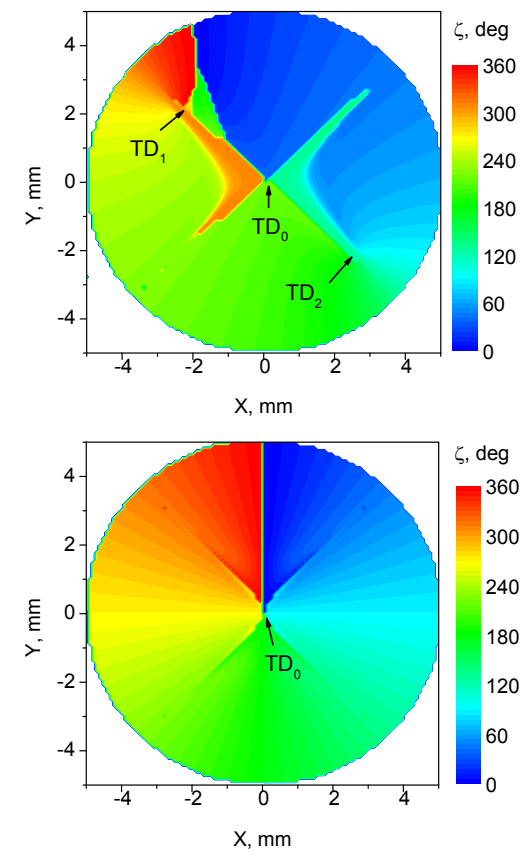

(a)

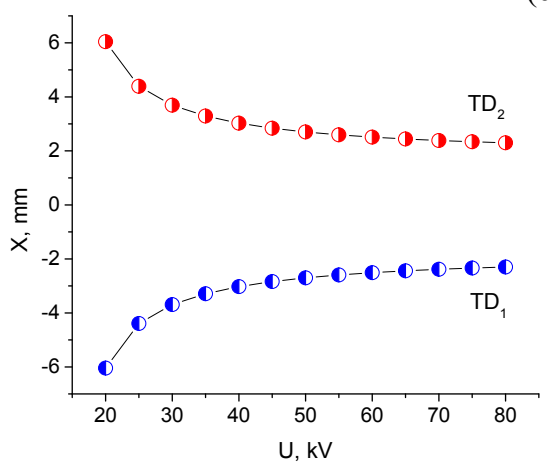

(c)

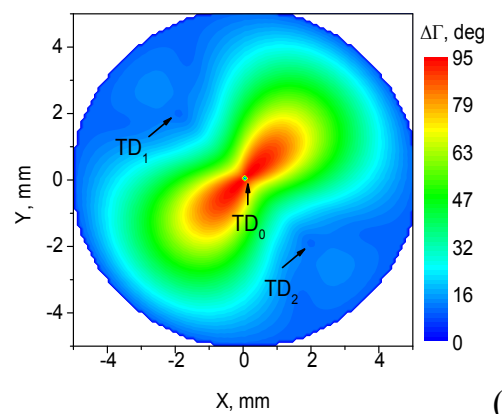

(b)

Fig. 3. Spatial maps of the angle of optical indicatrix orientation (a) and the phase difference (b) simulated for the crystals belonging to the point symmetry group 23 in the case when a conically shaped electric field $(U=40 \mathrm{kV})$ is applied along the [001] axis and light propagates in the same direction. Panel (c) shows map of the angle of optical indicatrix orientation obtained in the case of pure electrooptic Kerr effect $(U=10 \mathrm{kV})$.

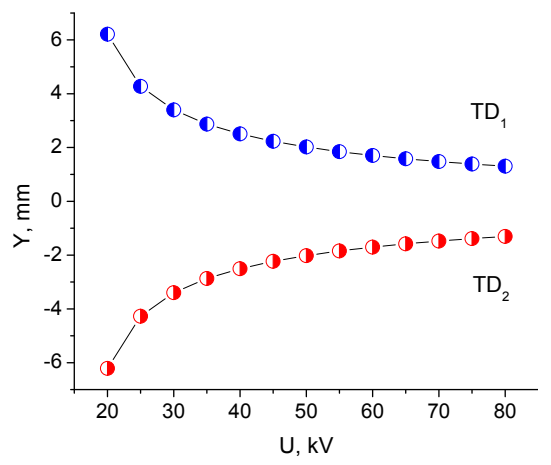

Fig. 4. Dependences of coordinates $X$ and $Y$ of the TDs on the electric voltage applied to the crystals that belong to the point symmetry group 23 .

In our recent work [2], we have partly analysed the behaviour of TDs of the optical indicatrix orientation under conically shaped electric field applied along the axis [001] in the crystals that belong to the point hexagonal symmetry group $\overline{6} \mathrm{~m} 2$. In that work we have revealed three lateral TDs and one central TD basing on purely analytical methods. However, we could not found a complete set of the corresponding solutions due to analytical difficulties. Therefore we perform here a more refined analysis. Let us consider the birefringence and the angle of optical indicatrix rotation within the uniform cell of a crystal:

$$
\begin{gathered}
\Delta n_{X Y}=-\frac{n_{o}^{3}}{2} \sqrt{\left[\left(R_{11}-R_{12}\right)\left(E_{1}^{2}-E_{2}^{2}\right)+2 r_{11} E_{1}\right]^{2}+4\left[R_{66} E_{1} E_{2}-2 r_{11} E_{2}\right]^{2}}, \\
\tan 2 \zeta_{Z}=\frac{2\left(R_{66} E_{1} E_{2}-2 r_{11} E_{2}\right)}{2 r_{11} E_{1}+R_{66}\left(E_{1}^{2}-E_{2}^{2}\right)},
\end{gathered}
$$

with the electrooptic coefficients $r_{11}=2 \times 10^{-12} \mathrm{~m} / \mathrm{V}$ and $R_{66}=2 \times 10^{-18} \mathrm{~m}^{2} / \mathrm{V}^{2}$ $\left(R_{66}=R_{11}-R_{12}\right)$, and the ordinary refractive index $n_{o}=1.7$. Then one can form the system of 
equations

$$
\left\{\begin{array}{l}
R_{66} E_{1} E_{2}-2 r_{11} E_{2}=0 \\
2 r_{11} E_{1}+R_{66}\left(E_{1}^{2}-E_{2}^{2}\right)=0
\end{array} .\right.
$$

Eqs. (15) result in seven solutions rather than four as concluded in Ref. [2]. They can be obtained numerically. Namely, one arrives at one central $\mathrm{TD}_{0}$ with the coordinates $X=Y=0$ and the strength $-1 / 2$, as well as three pairs of the lateral TDs with the strength modules $|1 / 2|$ and the opposite strength signs within the pairs (see Fig. 5), which fully comprise the earlier solutions obtained in the work [2].

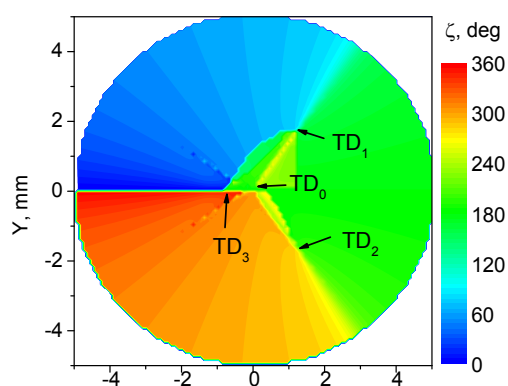

$\mathrm{X}, \mathrm{mm}$

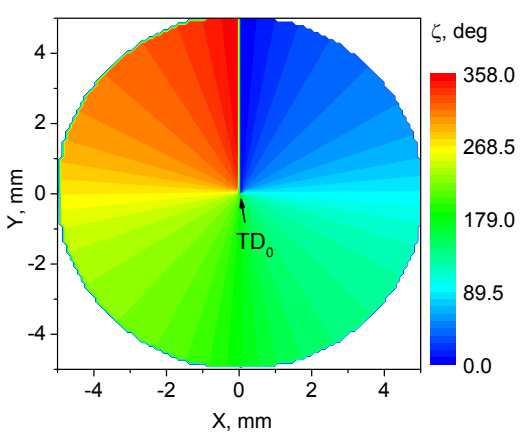

(a)

(c)

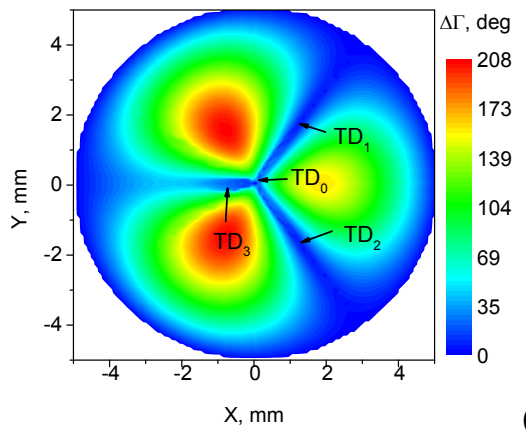

(b)

Fig. 5. Spatial maps of the angle of optical indicatrix orientation (a) and the phase difference (b) simulated for the crystals belonging to the point symmetry group $\overline{6} \mathrm{~m} 2$ in the case when a conically shaped electric field $(U=35.1 \mathrm{kV})$ is applied along the [001] axis and light propagates in the same direction. Panel (c) shows map of the angle of optical indicatrix orientation obtained in the case of pure electrooptic Kerr effect $(U=10 \mathrm{kV})$.

A birth of the pairs of TDs occurs due to the topological reaction $0=p_{\mathrm{TD}^{*} i}+p_{\mathrm{TD} i}=$ $-1 / 2+1 / 2$, where $p_{\mathrm{TD} i}$ implies the topological strength of the $i$ th TD. The changes in the coordinates of these lateral defects $\left(\mathrm{TD}_{1}\right.$ and $\mathrm{TD}_{1}^{*}, \mathrm{TD}_{2}$ and $\mathrm{TD}_{2}$, and $\mathrm{TD}_{3}$ and $\mathrm{TD}_{3}$ ) occurring with varying voltage are displayed in Fig. 6. It is seen from Fig. 6 that the three lateral defects, which have been omitted in the study [2] $\left(\mathrm{TD}^{*}, \mathrm{TD}_{2}{ }_{2}\right.$ and $\mathrm{TD}_{3}$ ), move out of the beam aperture with increasing electric voltage. The behaviours of the other defects have been analyzed in detail in the work [2]. Nonetheless, here we are also to remind that, as the Pockels effect vanishes, the two TDs are added thus leading to appearance of the central defect with the strength of +1 $\left(p_{\mathrm{TD} 1}+p_{\mathrm{TD} 2}=+1 / 2+1 / 2=1\right)$, while the two other TDs annihilate: $p_{\mathrm{TD} 3}+p_{\mathrm{TD} 0}=+1 / 2-1 / 2=0$ (see Fig. $4 c)$.

Since the point symmetry group $\overline{6}$ is a subgroup of the group $\overline{6} \mathrm{~m} 2$, the behaviours of the TDs for those groups should be similar. For the group $\overline{6}$, the induced birefringence and the angle of optical indicatrix rotation within the uniform cell of a crystal are as follows:

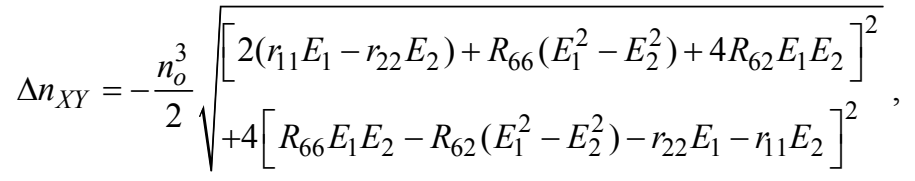




$$
\tan 2 \zeta_{Z}=\frac{2\left(R_{66} E_{1} E_{2}-R_{62}\left(E_{1}^{2}-E_{2}^{2}\right)-r_{22} E_{1}-r_{11} E_{2}\right)}{2\left(r_{11} E_{1}-r_{22} E_{2}\right)+R_{66}\left(E_{1}^{2}-E_{2}^{2}\right)+4 R_{62} E_{1} E_{2}},
$$

where we have taken the electrooptic parameters $r_{11}=1 \times 10^{-12} \mathrm{~m} / \mathrm{V}, \quad r_{22}=1.2 \times 10^{-12} \mathrm{~m} / \mathrm{V}$, $R_{66}=1.05 \times 10^{-18} \mathrm{~m}^{2} / \mathrm{V}^{2}$ and $R_{62}=1.1 \times 10^{-18} \mathrm{~m}^{2} / \mathrm{V}^{2}$, and the refractive index $n_{o}=1.7$.
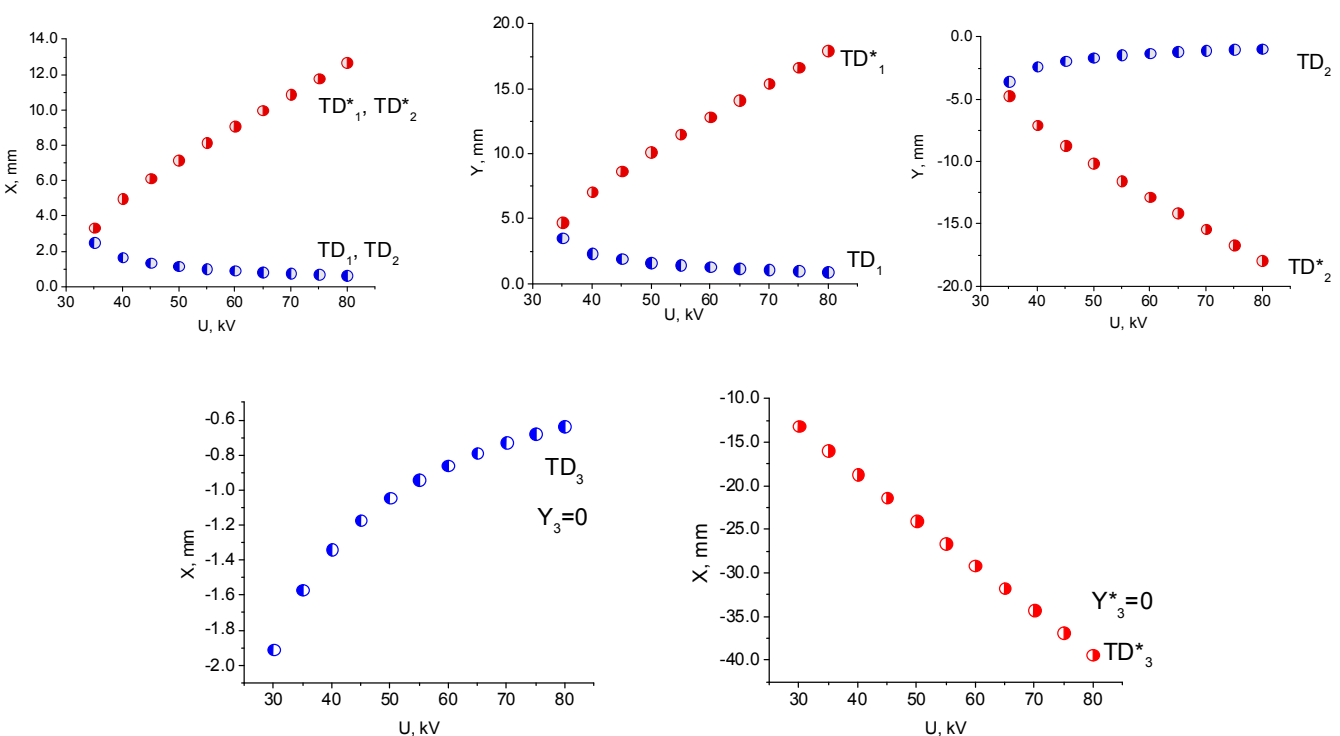

Fig. 6. Dependences of coordinates of the lateral TDs on the electric voltage applied to the crystals that belong to the point symmetry group $\overline{6} \mathrm{~m} 2$.
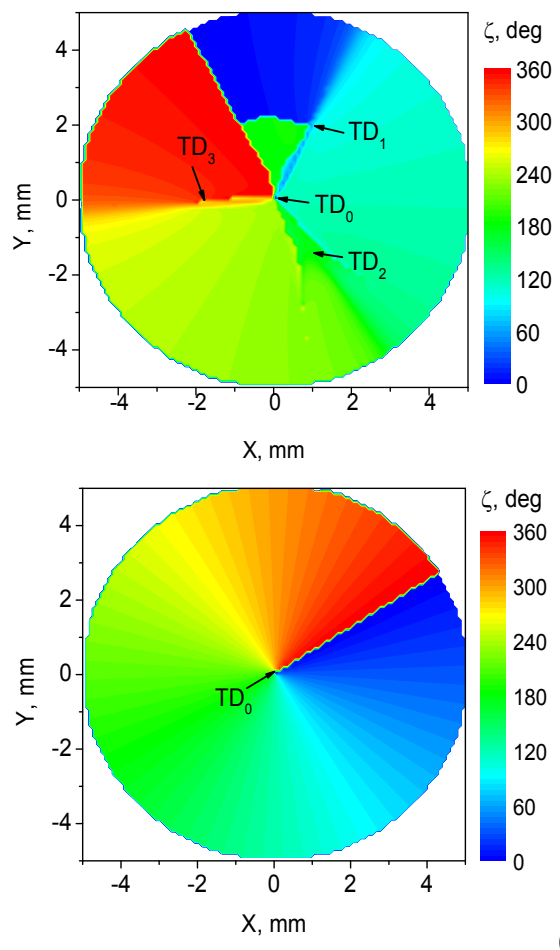

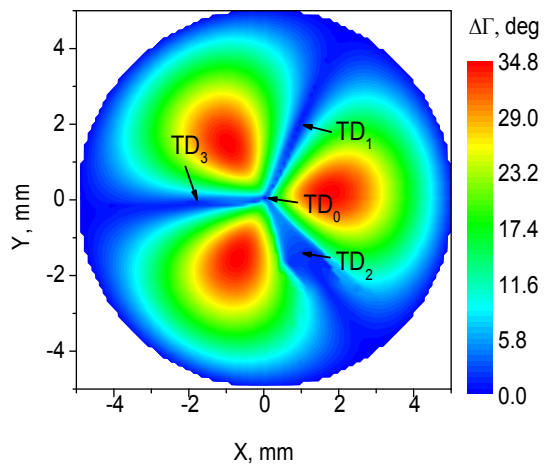

(b)

Fig. 7. Spatial maps of the angle of optical indicatrix orientation (a) and the phase difference (b) simulated for the crystals belonging to the point symmetry group $\overline{6}$ in the case when a conically shaped electric field $(U=15 \mathrm{kV})$ is applied along the [001] axis and light propagates in the same direction. Panel (c) shows map of the angle of optical indicatrix orientation obtained in the case of pure electrooptic Kerr effect $(U=10 \mathrm{kV})$.

(c) 
As seen from Fig. 7 and Fig. 8, seven TDs appear in the crystals that belong to the point group $\overline{6}$ under the conical electric field. These are a central $\mathrm{TD}_{0}$ with the strength equal to $-1 / 2$ and three pairs of lateral TDs $\left(\mathrm{TD}_{1}\right.$ and $\mathrm{TD}_{1}{ }_{1}, \mathrm{TD}_{2}$ and $\mathrm{TD}_{2}$, and $\mathrm{TD}_{3}$ and $\left.\mathrm{TD}_{3}{ }_{3}\right)$. As always, the phase difference is equal to zero in the TD cores. The strengths of the defects within the pairs are characterized by the same half-integer values and the opposite signs. These pairs appear due to the process of birth of the TDs. As the voltage increases, the defects TD $_{1,2,3}$ with the strengths $-1 / 2$ move out of the centre of the beam cross section, while $\mathrm{TD}_{1,2,3}$ approach the centre. It is interesting to notice that this movement is not radial as observed in our previous examples. The defects $\mathrm{TD}_{1,2,3}$ move tangentially with respect to $\mathrm{TD}_{1,2,3}$. As the Pockels effect becomes zero, the central defect changes its strength to +1 due to the topological reaction of adding and annihilation of TDs: $p_{\mathrm{TD} 0}+p_{\mathrm{TD} 1}+p_{\mathrm{TD} 2}+p_{\mathrm{TD} 3}=-1 / 2+1 / 2+1 / 2+1 / 2=+1$ (see Fig. $7 \mathrm{c}$ ).
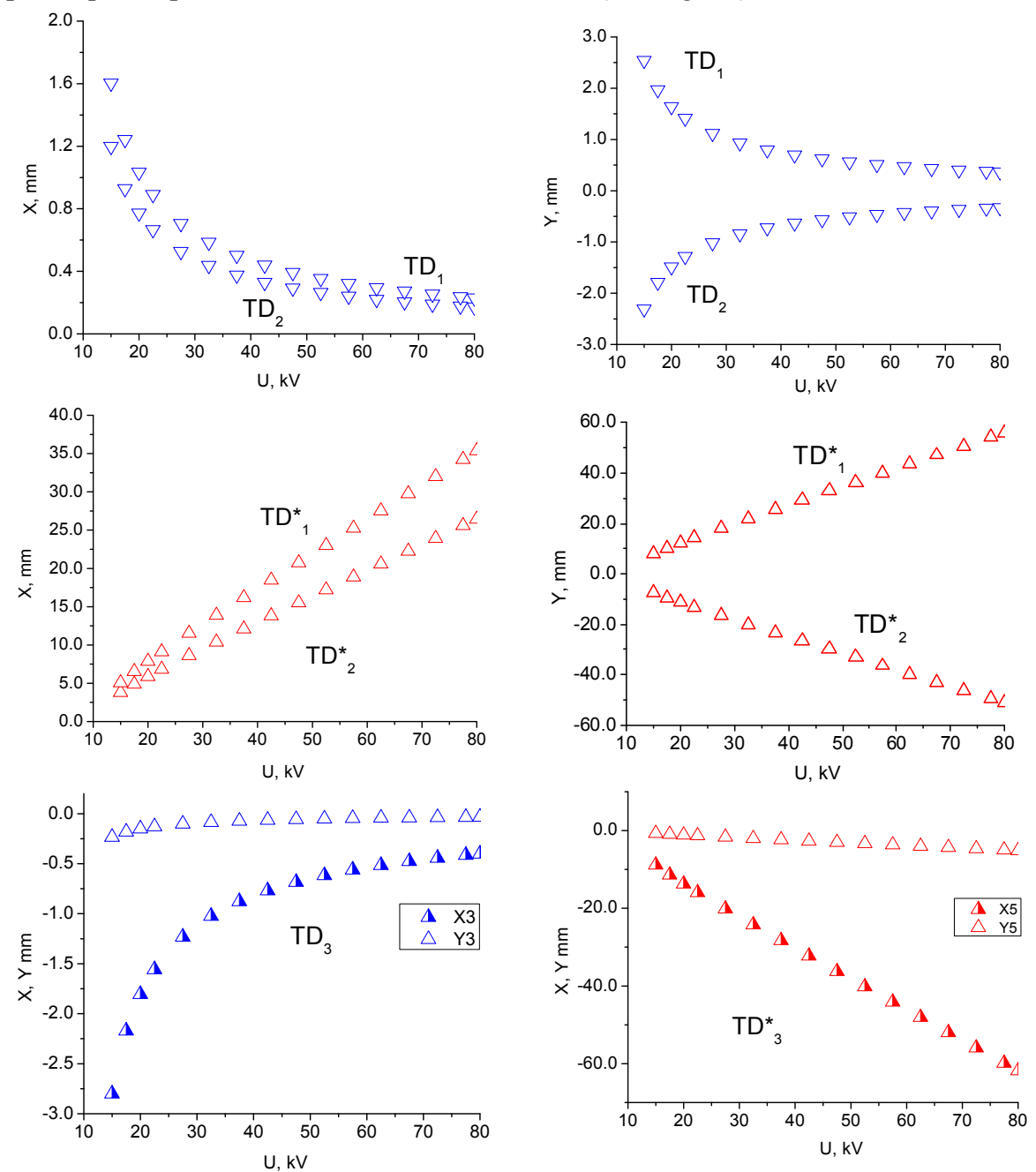

Fig. 8. Dependences of coordinates of the lateral TDs on the electric voltage applied to the crystals that belong to the point symmetry group $\overline{6}$.

Let us consider the behaviour of the TDs for the crystals belonging to the trigonal point symmetry groups 32 and $3 \mathrm{~m}$. These groups can be characterized with two different crystallographic settings. The two-fold axis in the group 32 can be parallel to either $X$ axis or $Y$ 
axis of the optical indicatrix. Likewise, the mirror plane in the group $3 \mathrm{~m}$ can be perpendicular to either $X$ axis or $Y$ axis. The relevant choice depends on the constitutive parameters of crystals, i.e. on the sign of piezoelectric coefficient $d_{33}$ or the sign of elastic compliance $S_{14}$ [9]. Moreover, the different settings are characterized by different structures of the third-rank polar tensor that describes the Pockels effect. As a consequence, the behaviours of the TDs for these setting can differ, too. We begin with the analysis for the crystallographic settings $32(2 \| X)$ and $3 \mathrm{~m}(\mathrm{~m} \perp Y)$. The relations for the induced birefringence and the angle of optical indicatrix orientation are as follows:

$$
\begin{gathered}
\Delta n_{X Y}=-\frac{n_{o}^{3}}{2} \sqrt{\left[2 r_{11} E_{1}+R_{66}\left(E_{1}^{2}-E_{2}^{2}\right)+2 R_{14} E_{3} E_{2}\right]^{2}+4\left[R_{14} E_{3} E_{1}+R_{66} E_{1} E_{2}-r_{11} E_{2}\right]^{2}}, \\
\tan 2 \zeta_{Z}=\frac{2\left(R_{14} E_{3} E_{1}+R_{66} E_{1} E_{2}-r_{11} E_{2}\right)}{2 r_{11} E_{1}+R_{66}\left(E_{1}^{2}-E_{2}^{2}\right)+2 R_{14} E_{3} E_{2}} .
\end{gathered}
$$

Here we use the parameter values $r_{11}=1 \times 10^{-12} \mathrm{~m} / \mathrm{V}, \quad R_{66}=1 \times 10^{-18} \mathrm{~m}^{2} / \mathrm{V}^{2}$, $R_{14}=0.1 \times 10^{-18} \mathrm{~m}^{2} / \mathrm{V}^{2}$, and $n_{o}=1.7$. The system of equations needed to obtain the coordinates of the TDs reads as

$$
\left\{\begin{array}{l}
R_{14} E_{3} E_{1}+R_{66} E_{1} E_{2}-r_{11} E_{2}=0 \\
2 r_{11} E_{1}+R_{66}\left(E_{1}^{2}-E_{2}^{2}\right)+2 R_{14} E_{3} E_{2}=0
\end{array} .\right.
$$
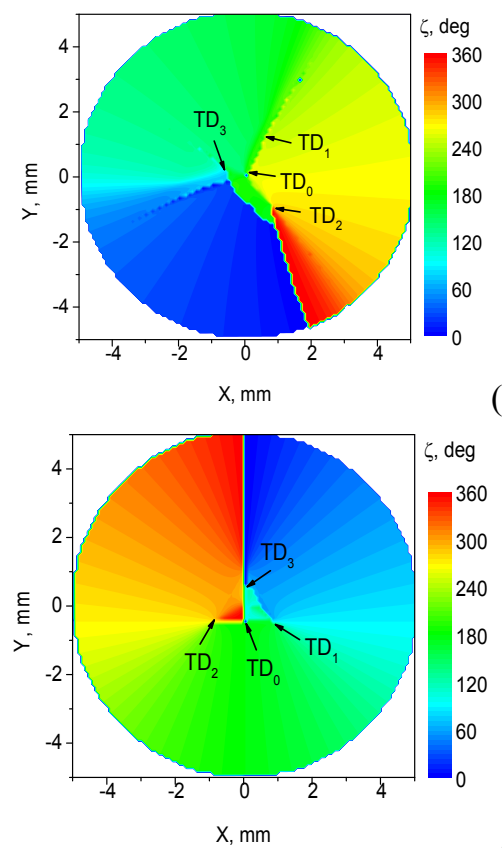

(a)

(c)

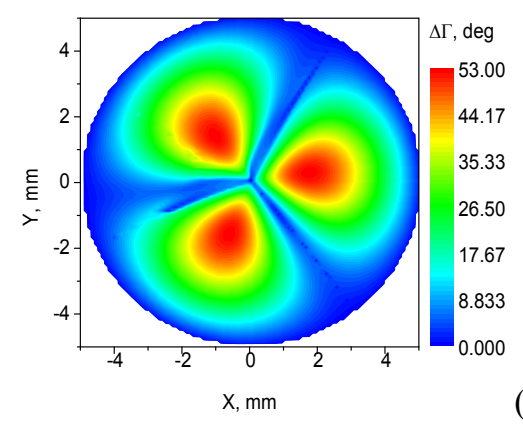

(b)

Fig. 9. Spatial maps of the angle of optical indicatrix orientation (a) and the phase difference (b) simulated for the crystals belonging to the point symmetry groups $32(2 \| X)$ and $3 \mathrm{~m}(\mathrm{~m} \perp \mathrm{Y})$ in the case when a conically shaped electric field $(U=30 \mathrm{kV})$ is applied along the [001] axis and light propagates in the same direction. Panel (c) shows map of the angle of optical indicatrix orientation obtained in the case of pure electrooptic Kerr effect $(U=10 \mathrm{kV})$.

Fig. 9 and Fig. 10 demonstrate that seven TDs appear under the conical electric field. These are a central $\mathrm{TD}_{0}$ with the strength $-1 / 2$ and three pairs of the lateral $\mathrm{TDs}\left(\mathrm{TD}_{1}\right.$ and $\mathrm{TD}^{*}{ }_{1}, \mathrm{TD}_{2}$ and $\mathrm{TD}_{2}{ }_{2}$, and $\mathrm{TD}_{3}$ and $\mathrm{TD}_{3}$ ). Their behaviour resembles that peculiar for the symmetry group $\overline{6} \mathrm{~m} 2$ : the pairs of defects appear due to the birth process, while the central defect exists due to axial symmetry of the conical field. When the Pockels effect vanishes, four defects with half-integer strengths are available, namely the central TD and the three lateral ones (see Fig. 9c). These 
defects do not change their positions when the voltage increases. The appearance of four TDs with half-integer strengths, instead of one central defect with the unit strength, can be explained by relatively low symmetry of the trigonal crystals. This leads to a necessity for division of the central defect as soon as the conical field is applied.
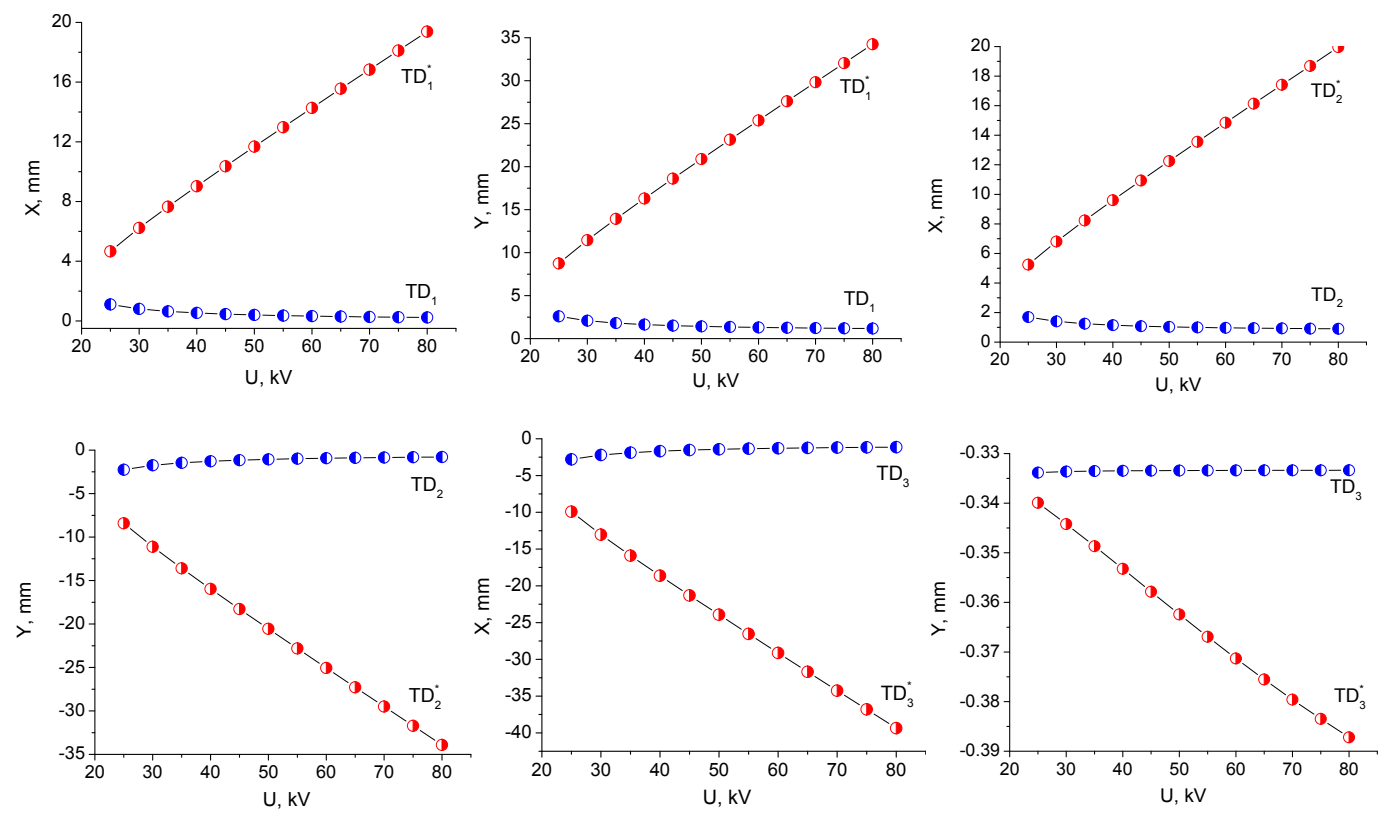

Fig. 10. Dependences of coordinates of the lateral TDs on the electric voltage applied to the crystals that belong to the point symmetry groups $32(2 \| X)$ and $3 m(m \perp Y)$.

Now we write out the induced birefringence, the angle of optical indicatrix orientation and the system of equations that defines the coordinates of the TDs for the crystals belonging to the crystallographic settings $32(2 \| Y)$ and $3 \mathrm{~m}(\mathrm{~m} \perp X)$ :

$$
\begin{gathered}
\Delta n_{X Y}=-\frac{n_{o}^{3}}{2} \sqrt{\left[2 R_{14} E_{3} E_{2}+R_{66}\left(E_{1}^{2}-E_{2}^{2}\right)-2 r_{22} E_{2}\right]^{2}+4\left[R_{14} E_{3} E_{1}+R_{66} E_{1} E_{2}-r_{22} E_{1}\right]^{2}}, \\
\tan 2 \zeta_{Z}=\frac{2\left(R_{14} E_{3} E_{1}+R_{66} E_{1} E_{2}-r_{22} E_{1}\right)}{2 R_{14} E_{3} E_{2}+R_{66}\left(E_{1}^{2}-E_{2}^{2}\right)-2 r_{22} E_{2}}, \\
\left\{\begin{array}{l}
R_{14} E_{3} E_{1}+R_{66} E_{1} E_{2}-r_{22} E_{1}=0 \\
2 R_{14} E_{3} E_{2}+R_{66}\left(E_{1}^{2}-E_{2}^{2}\right)-2 r_{22} E_{2}=0
\end{array}\right.
\end{gathered}
$$

Here we put $r_{22}=1 \times 10^{-12} \mathrm{~m} / \mathrm{V}, \quad R_{66}=1.5 \times 10^{-18} \mathrm{~m}^{2} / \mathrm{V}^{2}$ and $R_{14}=0.5 \times 10^{-18} \mathrm{~m}^{2} / \mathrm{V}^{2}$, and $n_{o}=1.7$. Eqs. (23) result in seven solutions: a central defect $\mathrm{TD}_{0}$ and three pairs of the lateral defects $\left(\mathrm{TD}_{1}\right.$ and $\mathrm{TD}_{1}{ }_{1}, \mathrm{TD}_{2}$ and $\mathrm{TD}_{2}{ }_{2}$, and $\mathrm{TD}_{3}$ and $\left.\mathrm{TD}_{3}\right)$. While the central $\mathrm{TD}$ has the strength-1/2 (see Fig. 11a-d), the strengths of the lateral TDs are half-integer and their signs are opposite within the pairs (see Fig. 11e-h).

The TDs with the strength $-1 / 2$, which are located further away from the centre (i.e., the defects $\mathrm{TD}^{*}{ }_{1}, \mathrm{TD}_{2}{ }_{2}$ and $\mathrm{TD}_{3}$ ), leave the beam cross section with increasing voltage. On the contrary, the defects which are located closer to the centre and have the same strength of $+1 / 2$ $\left(\mathrm{TD}_{1}, \mathrm{TD}_{2}\right.$ and $\mathrm{TD}_{3}$ ) move towards the central $\mathrm{TD}$. At the voltage equal to $\sim 9.8 \mathrm{kV}$ they reach the 

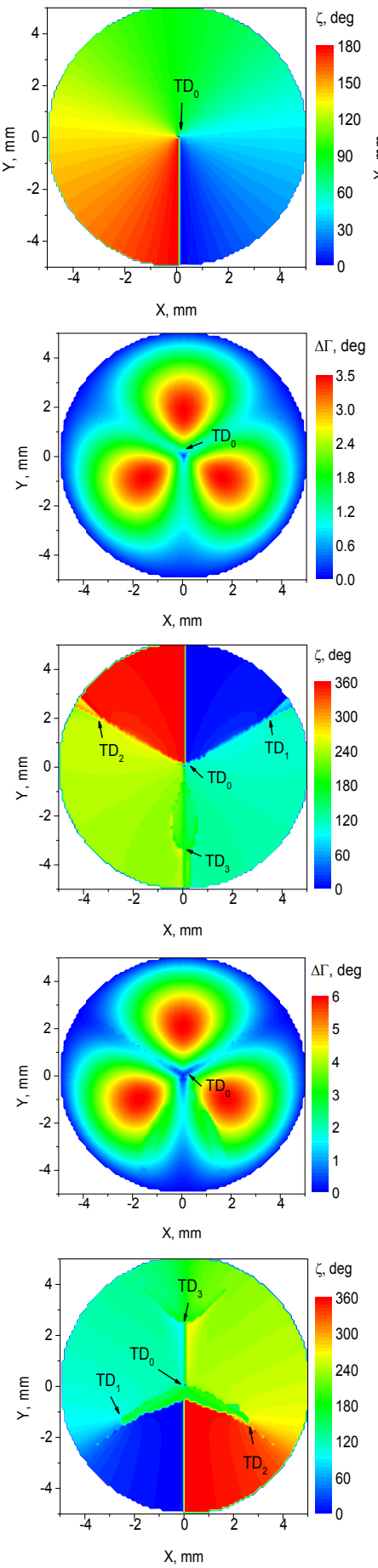
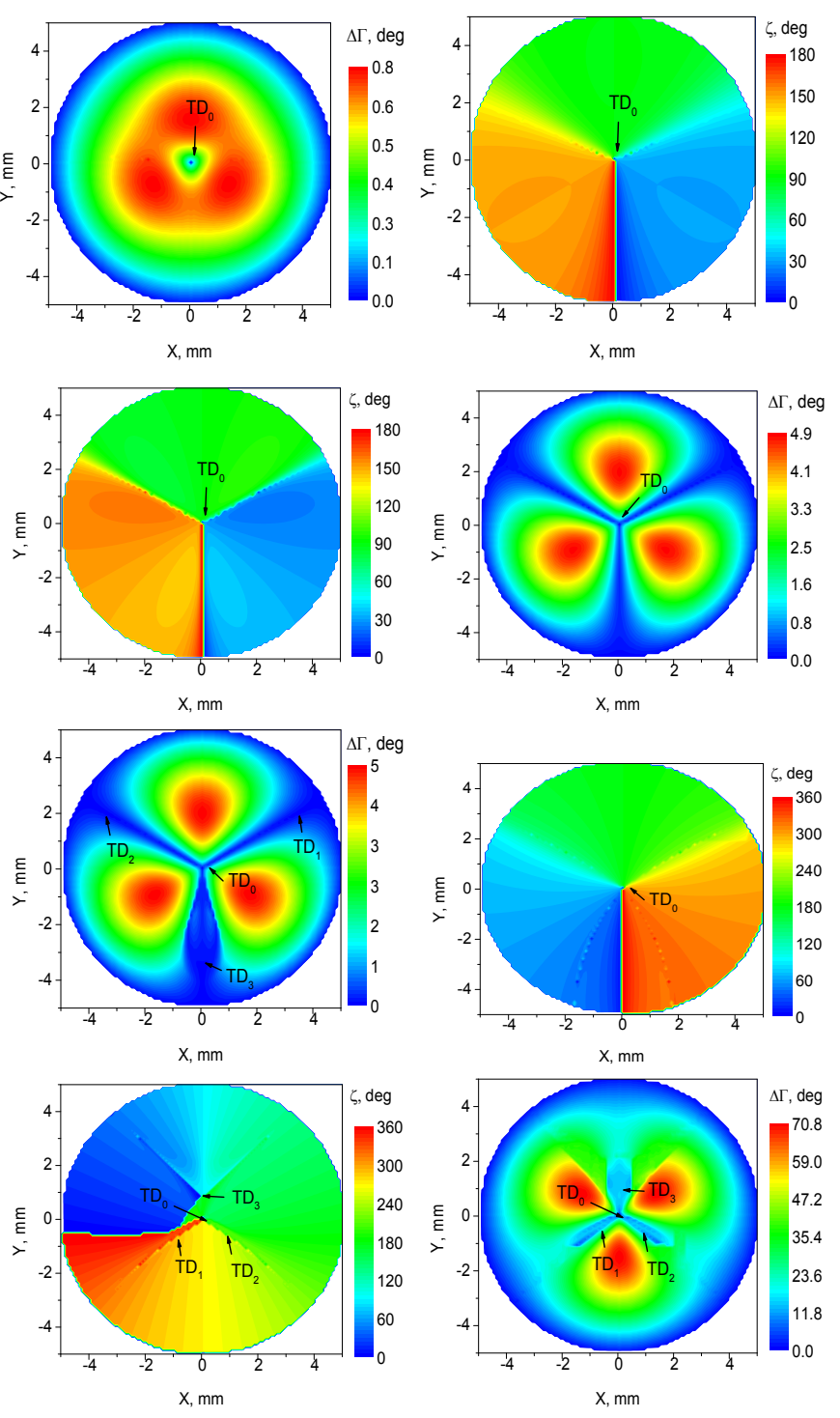

Fig. 11. Spatial maps of the angle of optical indicatrix orientation (a, e, c, g, i, k) and the phase difference (b, d, f, h, j, I) simulated for the crystals belonging to the point symmetry groups $32(2 \| Y)$ and $3 \mathrm{~m}(\mathrm{~m} \perp X)$ in the case when a conically shaped electric field is applied along the [001] axis and light propagates in the same direction: $1 \mathrm{kV}(\mathrm{a}, \mathrm{b}), 5 \mathrm{kV}(\mathrm{c}, \mathrm{d}), 7 \mathrm{kV}(\mathrm{e}, \mathrm{f}), 7.5 \mathrm{kV}(\mathrm{g}, \mathrm{h}) 9 \mathrm{kV}(\mathrm{i}$, $\mathrm{j})$, and $30 \mathrm{kV}(\mathrm{k}, \mathrm{l})$. Panel $(\mathrm{m})$ shows map of the angle of optical indicatrix orientation obtained in the case of pure electrooptic Kerr effect $(U=10 \mathrm{kV})$.

centre of the beam cross section, thus creating the central TD with the strength equal to +1 : $p_{\mathrm{TD} 0}+p_{\mathrm{TD} 1}+p_{\mathrm{TD} 2}+p_{\mathrm{TD} 3}=-1 / 2+1 / 2+1 / 2+1 / 2=+1$. This process is accompanied by addition of the two defects and annihilation of the remaining two. When the voltage increases still more, one observes the process of division of the central TD. Then the central TD is divided into the four defects (see Fig. 11k, 1), the central one and the three lateral, with the strengths equal to $+1 / 2$. The latter lateral TDs move gradually out of the centre with increasing voltage (see Fig. 12). Finally, 
four defects are available when the Pockels coefficients become zero, their strengths being halfinteger. These are the central TD and the three lateral defects (see Fig. 11m). These defects do not change their positions with increasing voltage. Again, they appear due to relatively low symmetry of the trigonal crystals, which stipulates division of the central defect as soon as the conically shaped electric field is applied.
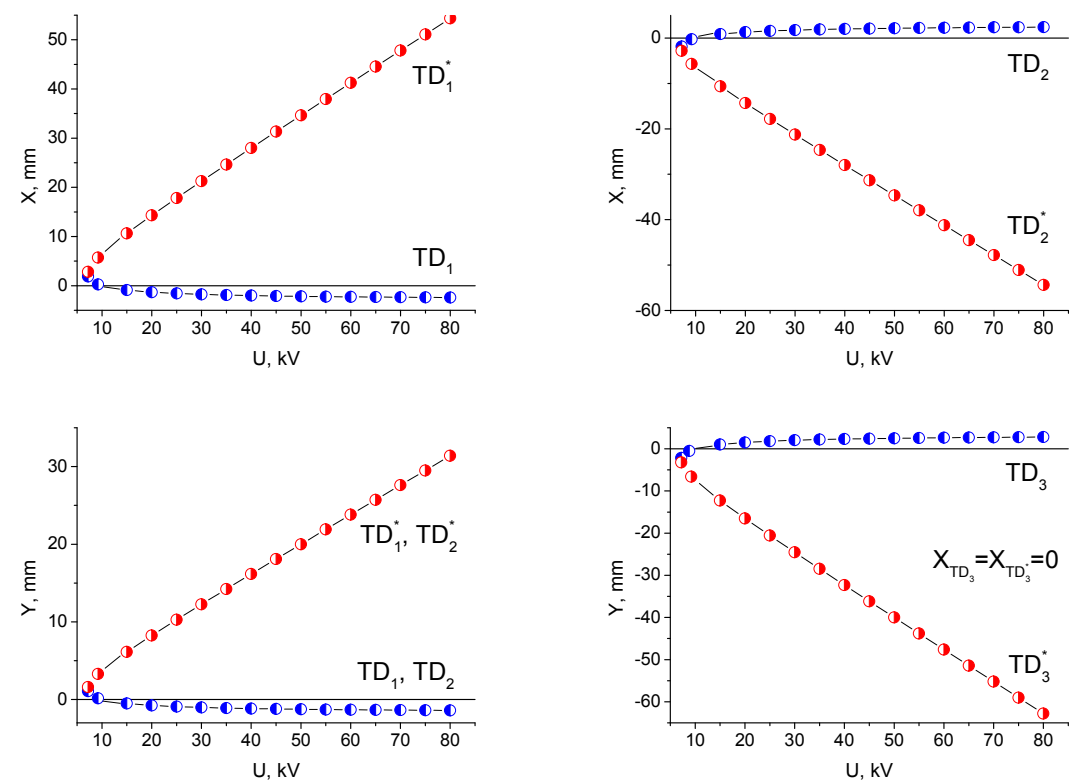

Fig. 12. Dependences of coordinates of the lateral TDs on the electric voltage applied to the crystals that belong to the point symmetry groups $32(2 \| Y)$ and $3 m(m \perp X)$.

Now let us consider the last example, the crystals belonging to the point symmetry group 3 . The appropriate relations for the birefringence and the angle of optical indicatrix rotation are as follows:

$$
\begin{aligned}
& \Delta n_{X Y}=-\frac{n_{o}^{3}}{2} \sqrt{\begin{array}{l}
\left.2\left(r_{11} E_{1}-r_{22} E_{2}\right)+R_{66}\left(E_{1}^{2}-E_{2}^{2}\right)+2 R_{14} E_{3} E_{2}-2 R_{25} E_{3} E_{1}+4 R_{62} E_{1} E_{2}\right]^{2} \\
+4\left[R_{62}\left(E_{2}^{2}-E_{1}^{2}\right)+R_{25} E_{2} E_{3}+R_{14} E_{3} E_{1}+R_{66} E_{1} E_{2}+r_{22} E_{1}-r_{11} E_{2}\right]^{2}
\end{array}} \\
& \tan 2 \zeta_{Z}=\frac{2\left(R_{62}\left(E_{2}^{2}-E_{1}^{2}\right)+R_{25} E_{2} E_{3}+R_{14} E_{3} E_{1}+R_{66} E_{1} E_{2}+r_{22} E_{1}-r_{11} E_{2}\right)}{2\left(r_{11} E_{1}-r_{22} E_{2}\right)+R_{66}\left(E_{1}^{2}-E_{2}^{2}\right)+2 R_{14} E_{3} E_{2}-2 R_{25} E_{3} E_{1}+4 R_{62} E_{1} E_{2}},
\end{aligned}
$$

where $r_{11}=10^{-12} \mathrm{~m} / \mathrm{V}, r_{22}=0.5 \times 10^{-12} \mathrm{~m} / \mathrm{V}, R_{66}=1.05 \times 10^{-18} \mathrm{~m}^{2} / \mathrm{V}^{2}, R_{25}=0.5 \times 10^{-18} \mathrm{~m}^{2} / \mathrm{V}^{2}$, $R_{14}=1 \times 10^{-18} \mathrm{~m}^{2} / \mathrm{V}^{2}, R_{62}=1.1 \times 10^{-18} \mathrm{~m}^{2} / \mathrm{V}^{2}$ and $n_{o}=1.7$.

Solving the system of equations

$$
\left\{\begin{array}{l}
R_{62}\left(E_{2}^{2}-E_{1}^{2}\right)+R_{25} E_{2} E_{3}+R_{14} E_{3} E_{1}+R_{66} E_{1} E_{2}+r_{22} E_{1}-r_{11} E_{2}=0 \\
2\left(r_{11} E_{1}-r_{22} E_{2}\right)+R_{66}\left(E_{1}^{2}-E_{2}^{2}\right)+2 R_{14} E_{3} E_{2}-2 R_{25} E_{3} E_{1}+4 R_{62} E_{1} E_{2}=0
\end{array},\right.
$$

one can arrive at seven solutions: a central TD and three pairs of the lateral $\mathrm{TDs}\left(\mathrm{TD}_{1}\right.$ and $\mathrm{TD}^{*}$, $\mathrm{TD}_{2}$ and $\mathrm{TD}_{2}^{*}$, and $\mathrm{TD}_{3}$ and $\mathrm{TD}_{3}{ }_{3}$ ). The central $\mathrm{TD}$ has the strength $-1 / 2$ (see Fig. 13a) and the strengths of the lateral defects are also half-integer. As always, the signs of those TDs are opposite within the pairs (see Fig. 13a). The above TD pairs appear due to the process of defect birth 
analyzed above. The defects of the strength $-1 / 2$, which are located further away from the centre $\left(\mathrm{TD} *{ }_{1}, \mathrm{TD}^{*}{ }_{2}\right.$ and $\mathrm{TD}{ }_{3}$ ), leave the beam cross section with increasing voltage (see Fig. 14). At the same time, the corresponding defects located closer to the centre $\left(\mathrm{TD}_{1}, \mathrm{TD}_{2}\right.$ and $\left.\mathrm{TD}_{3}\right)$ 'oscillate' around their initial positions under the same condition. Zeroing of the Pockels coefficients in Eqs. (24)-(26) implies that the system of Eqs. (26) has only four solutions corresponding to the central TD (the strength equal to $-1 / 2$ ) and the three lateral defects with the strengths $+1 / 2$ (see Fig. 13b). This means that, in the hypothetical case of $r_{11}=r_{22}=0$, the central defect of the integer strength available in the crystals belonging to the symmetry group 3 is always divided into four defects with half-integer strengths.
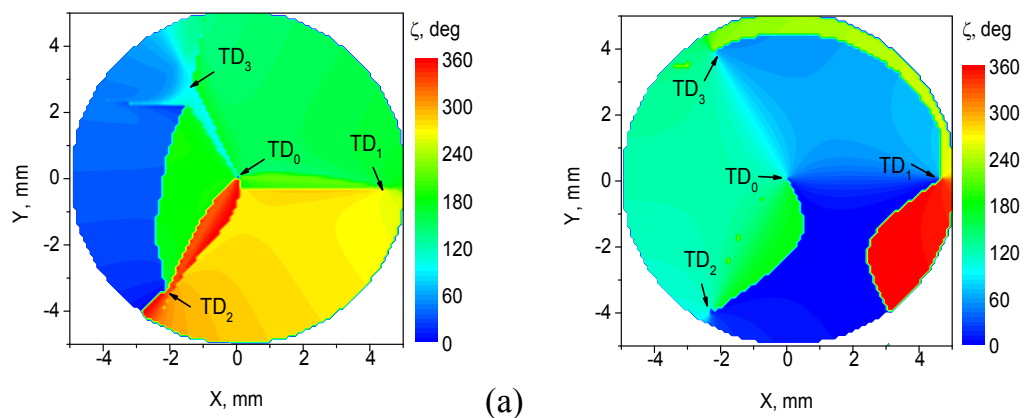

(a)

(b)

Fig. 13. Spatial maps of the angle of optical indicatrix orientation simulated for the crystals belonging to the point symmetry group 3 in the case when a conically shaped electric field $(U=40 \mathrm{kV})$ is applied along the [001] axis and light propagates in the same direction: panels (a) corresponds to the combined action of the electrooptic Pockels and Kerr effects and panel (b) to the pure Kerr effect $(U=10 \mathrm{kV})$.
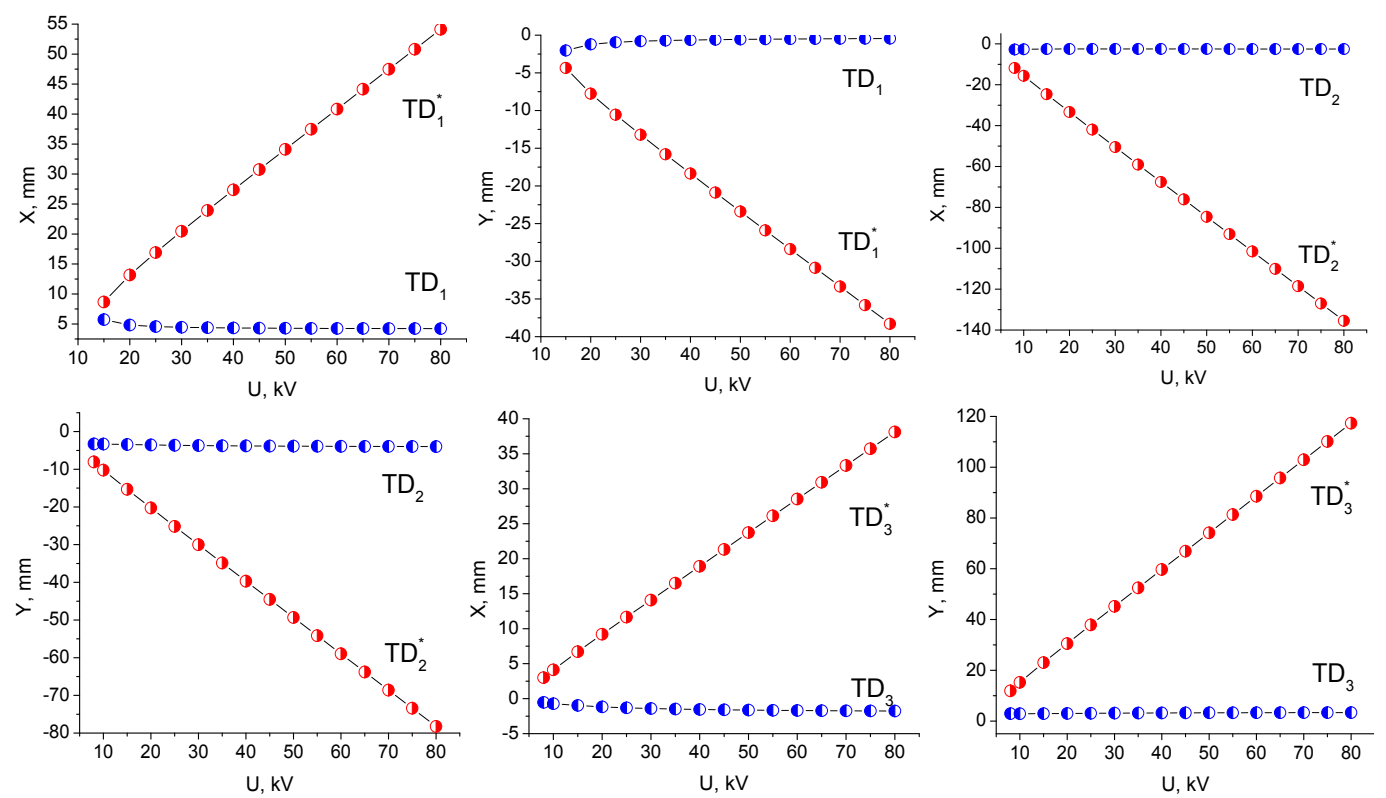

Fig. 14. Dependences of coordinates of the lateral TDs on the electric voltage applied to the crystals that belong to the point symmetry groups 3 .

\section{Conclusions}

We have studied the behaviour of TDs of the optical indicatrix orientation under the conditions when the electrooptic Pockels and Kerr nonlinearities coexist in the cubic, hexagonal, trigonal and tetragonal crystals and a conically shaped electric field is applied to those crystals. This is 
continuation of the studies initiated in Ref. [1]. The processes of birth, addition, division and annihilation of the TDs of optical indicatrix orientation are observed under varying electric field. These processes are accompanied by the topological reactions at which the conservation law for the strength of TDs holds true.

In general, the behaviours of the TDs can be described by the following four scenarios.

1. In the crystals of the point symmetry groups $\overline{4} 3 \mathrm{~m}$ and 23 (the field direction [111]) and the point symmetry groups $\overline{6} \mathrm{~m} 2, \overline{6}$ and $32(2 \| X)$ and $3 \mathrm{~m}(\mathrm{~m} \perp Y)$ (the field direction [001]), the electric field gives rise to a birth of three pairs of lateral TDs and appearance of a single central TD. The lateral defects are characterized by half-integer strength values and the opposite signs of those strengths within the pairs. The central defect has half-integer strength, too. When the electric voltage increases, the lateral TDs move in different manners: three of those TDs move out of the beam cross section and the remaining three towards the centre of the beam. When the Pockels coefficients of the crystals belonging to the symmetry groups $\overline{4} 3 \mathrm{~m}, 23, \overline{6} \mathrm{~m} 2$ and $\overline{6}$ tend to zero, the latter defects are added or annihilate pairwise, with consequent appearance of a central defect having the integer strength equal to one. On the other hand, zeroing of the Pockels coefficients in the crystals that belong to the symmetry groups $32(2 \| X)$ and $3 \mathrm{~m}(\mathrm{~m} \perp Y)$ gives rise to four TDs having half-integer strengths (a central TD and three lateral TDs). These defects do not change their positions with increasing voltage. They exist due to relatively low symmetry of the trigonal crystals.

2. In the crystals belonging to the point symmetry groups $\overline{4} 3 \mathrm{~m}, 23$ and $\overline{4}$, two lateral defects with half-integer strengths exist under conical electric field applied along the [001] direction. In the presence of both the Pockels and Kerr effects they have the same strength signs. These defects move towards the centre of the beam cross section with increasing voltage, though they reach it only when the Pockels coefficients become zero. In the latter case, due to the topological reaction of adding of TDs, the central defect acquires the integer topological strength equal to one.

3. In the crystals belonging to the point symmetry groups $32(2 \| Y)$ and $3 \mathrm{~m}(\mathrm{~m} \perp X)$, the electric field imposes a birth of three pairs of TDs and, additionally, a single central TD exists. The lateral defects are characterized with half-integer strength values and the opposite strength signs within the pairs. The central TD has also half-integer strength. Like in one of the cases described above, increasing voltage induces a complex motion of the lateral defects: three of them move out of the beam cross section and three towards the beam centre. It is important that, at some specific electric voltage, they reach the centre of the beam cross section, thus creating the central TD with the strength equal to +1 . The explanation of this effect is given by the topological reaction $p_{\mathrm{TD} 0}+p_{\mathrm{TD} 1}+p_{\mathrm{TD} 2}+p_{\mathrm{TD} 3}=-1 / 2+1 / 2+1 / 2+1 / 2=+1$. In this process the two defects are added together and the other two annihilate. When the voltage increases further on, a process of division of the central TD is observed: it is divided into four defects, a 'new' central one and three lateral TDs having the strengths $+1 / 2$. These lateral defects move gradually out of the centre with increasing voltage. Disappearance of the Pockels effect imposes appearance of four defects with half-integer strengths, a central TD and three lateral ones. These defects do not change their positions with increasing voltage.

4. In the crystals belonging to the point symmetry group 3, conically shaped electric field leads to appearance of seven TDs of optical indicatrix orientation: a central defect and three pairs of the lateral TDs $\left(\mathrm{TD}_{1}\right.$ and $\mathrm{TD}_{1}, \mathrm{TD}_{2}$ and $\mathrm{TD}_{2}{ }_{2}$, and $\mathrm{TD}_{3}$ and $\mathrm{TD}_{3}{ }_{3}$. The central $\mathrm{TD}$ has the strength $-1 / 2$ and the strength magnitude of the lateral TDs are half-integer, with the opposite signs within the pairs. The lateral pairs of TDs appear due to the topological reaction of defect 
birth, while the central one arises due to axial symmetry of the conical electric field. With increasing voltage the defects with the strengths $-1 / 2$, which are located further away from the centre $\left(\mathrm{TD}_{1}{ }_{1}, \mathrm{TD}_{2}{ }_{2}\right.$ and $\mathrm{TD}_{3}$ ), leave the beam cross section. On the other hand, the similar TDs closer to the centre $\left(\mathrm{TD}_{1}, \mathrm{TD}_{2}\right.$, and $\left.\mathrm{TD}_{3}\right)$ 'oscillate' around their initial positions with increasing electric voltage. When the Pockels coefficients tend to zero, four defects come to existence. These are a central TD with the strength $-1 / 2$ and three lateral TDs with the strengths $+1 / 2$. These defects appear due to the topological reaction of division. Namely, the central defect with the integer strength is divided into the four defects with half-integer strengths as soon as the conical field is applied. The latter process is conditioned by low enough symmetry of the trigonal crystals.

\section{References}

1. Vasylkiv Yu, Kryvyy T, Skab I and Vlokh R, 2014. Behaviour of topological defects of optical indicatrix orientation in cubic single crystals under conically distributed electric field. 1. The electric field and the optical beam parallel to the three-fold symmetry axis. Ukr. J. Phys. Opt. 15: 184-194.

2. Vasylkiv Yu, Skab I and Vlokh R, 2014. Crossover regime of optical vortices generation via electro-optic nonlinearity: the problem of optical vortices with the fractional charge generated by crystals. J. Opt. Soc. Amer. A. 31: 1936-1945.

3. Vlokh O G and Zheludev I S, 1960. Change of optical indicatrices of crystals under application of electric field. Kristallografiya. 5: 390-402.

4. Marrucci L, Manzo C and Paparo D, 2006. Optical spin-to-orbital angular momentum conversion in inhomogeneous anisotropic media. Phys. Rev. Lett. 96: 163905.

5. Skab I, Vasylkiv Yu, Savaryn V and Vlokh R, 2011. Optical anisotropy induced by torsion stresses in $\mathrm{LiNbO}_{3}$ crystals: appearance of an optical vortex. J. Opt. Soc. Amer. A. 28: 633640 .

6. Vasylkiv Yu, Skab I, Smyk M and Vlokh R, 2014. Topological defects of optical indicatrix orientation in stressed glasses: spatial distribution of optical anisotropy parameters. Appl. Opt. 53: 3967-3975.

7. Vlokh O G, 1965. Deformation of optical indicatrices at the quadratic and spontaneous electro-optical effects in crystals. Ukr. Fiz. Zhurn. X: 1001-1118.

8. Vasylkiv Yu, Skab I and Vlokh R, 2014. Double-charged optical vortices generation on the basis of electrooptic Kerr effect. Appl. Opt. 53: B60-B73.

9. Sirotin Yu I and Shaskolskaya M P. Fundamentals of crystal physics. Moscow: Nauka (1979).

Vasylkiv Yu., Kryvyy T., Skab I. and Vlokh R. 2015. Behaviour of topological defects of optical indicatrix orientation in cubic, hexagonal, trigonal and tetragonal crystals under conically distributed electric field. 2. The electric field and the optical beam parallel to the principal crystallographic directions. Ukr.J.Phys.Opt. 16: 1 - 16.

Анотація. У роботі досліджено поведінку топологічних дефектів (ТД) орієнтації оптичної індикатриси при співіснуванні електрооптичних нелінійностей керівського $i$ покельсівського типів у кристалах, що належать до кубічної, гексагональної, тригональної i тетрагональної сингоній, за умови прикладання конічного електричного поля вздовж головних кристалографічних і/або оптичних осей. Виявлено, щзо за згаданих умов реалізуються топологічні реакції, які супроводжуються народженням, додаванням, поділом $i$ анігіляцією ТД орієнтащії оптичної індикатриси. Показано, щуо за цих топологічних реакцій виконується закон збереження сили ТД, а поведінку ТД можна вичерпно описати чотирма різними сценаріями. 